\title{
Generation of recombinant MVA-norovirus: a comparison study of bacterial artificial chromosome- and marker-based systems
}

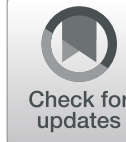

Franziska Kugler ${ }^{1}$, Ingo Drexler ${ }^{2}$, Ulrike Protzer ${ }^{1}$, Dieter Hoffmann ${ }^{1 *}$ and Hassan Moeini ${ }^{1 *}$

\begin{abstract}
Background: Recombinant Modified Vaccinia Virus Ankara has been employed as a safe and potent viral vector vaccine against infectious diseases and cancer. We generated recMVAs encoding norovirus Gll.4 genotype capsid protein by using a marker-based approach and a BAC-based system. In the marker-based approach, the capsid gene together with a reporter gene was introduced into the MVA genome in DF-1 cells. Several rounds of plaque purification were carried out to get rid of the WT-MVA. In the BAC-based approach, recMVA-BAC was produced by en passant recombineering in E. coli. Subsequently, the recMVAs were rescued in DF-1 cells using a helper rabbit fibroma virus. The BAC backbone and the helper virus were eliminated by passaging in DF-1 cells. Biochemical characteristics of the recMVAs were studied.

Results: We found the purification of the rare spontaneous recombinants time-consuming in the marker-based system. In contrast, the BAC-based system rapidly inserted the gene of interest in E. coli by en passant recombineering before virion production in DF-1 cells. The elimination of the reporter gene was found to be faster and more efficient in the BAC-based approach. With Western blotting and electron microscopy, we could prove successful capsid protein expression and proper virus-assembly, respectively. The MVA-BAC produced higher recombinant virus titers and infected DF-1 cells more efficiently.
\end{abstract}

Conclusions: Comparing both methods, we conclude that, in contrast to the tedious and time-consuming traditional method, the MVA-BAC system allows us to quickly generate high titer recMVAs.

Keywords: Bacterial artificial chromosome, Recombinant MVA, Self-excising, Norovirus

\section{Background}

Norovirus is highly infectious and can be serious to individuals with underlying conditions, the elderly, young children and immunocompromised patients. GII.4 genotype evolves faster than other genotypes and global pandemic outbreaks often correlate to the emergence of new GII.4 variants [1-3]. Major capsid protein (VP1) is responsible for virus attachment to the histo-blood group antigen [4]. The capsid protein is divided into shell (S) domain and a protruding $(\mathrm{P})$ domain linked together by a hinge of eight amino acids $[4,5]$. The aim of this study was to generate a recMVA expressing norovirus (NoV) capsid protein of GII.4 genotype. We plan to apply the constructs as

\footnotetext{
*Correspondence: dieter.hoffmann@tum.de; hassan.moeini@tum.de ${ }^{1}$ Institute of Virology, Faculty of Medicine, Technische Universität München, Munich, Germany

Full list of author information is available at the end of the article
}

vaccine candidates against this most prevalent gastroenteritis virus.

Modified Vaccinia Ankara (MVA) is a highly attenuated strain of vaccinia virus derived by extensive serial passages in chicken embryo fibroblasts (CEF) [6]. MVA can be produced in large scale in chicken cell lines under bio safety level 1 . Temporal high expression of MVA-delivered antigens can modulate antigen-specific immune responses, making it a suitable vaccine carrier [6-9]. We followed a traditional marker-based method (as reviewed by Drexler et al. [7]) and a BAC-based approach as described by Dai [10] and Cottingham et al. [11] for generation of recMVA-NoV constructs. Both techniques produced recombinant MVA delivering the desired antigen.

In the marker-based approach, a shuttle vector harboring a fluorescence reporter gene, the gene of interest 
(GOI) is inserted downstream of the modified $\mathrm{H} 5$ (mH5) promoter [12]. In wild-type MVA-infected cells, the gene of interest together with the reporter gene is introduced into the viral genome by homologous recombination (Fig. 1). Plaque purification is carried out to eventually get rid of the WT-MVA. To this end, fluorescent plaques are picked and tested for the presence of WT-MVA by PCR. As homologous recombination is not 100\% efficient, both of WT- and rec-MVA viral particles are released in the supernatant (Fig. 1b) and therefore, several rounds of plaque purification is required to eradicate the WT virus. The reporter gene will be eliminated from the MVA genomes gradually, as it is flanked by homologous regions that can recombine during replication. Selection for infected cells is difficult, as DF-1 cells do not form morphologic distinct plaques.
In the BAC-based system, recMVA is produced by en passant recombineering as previously described by Dai $[10,11]$. Red-mediated modification is performed in the E. coli strain GS1783 containing a temperaturedependent expression cassette for the recombination proteins and an L-arabinose-inducible I-SecI gene. In the first step, as shown in Fig. 2a, the gene of interest is introduced into a shuttle vector which is equipped with homologous regions to the DelVI of the MVA genome in the MVA-BAC plasmid. After heat induction, the Red recombinase promotes the insertion of our gene of interest along with the Kanamycin (Kan) resistance cassette and the I-SceI restriction site in the MVA genome. Positive clones are selected on agar plate supplemented with kanamycin and after confirmation of the insertion, selected BAC plasmids are subjected to the 2nd Red

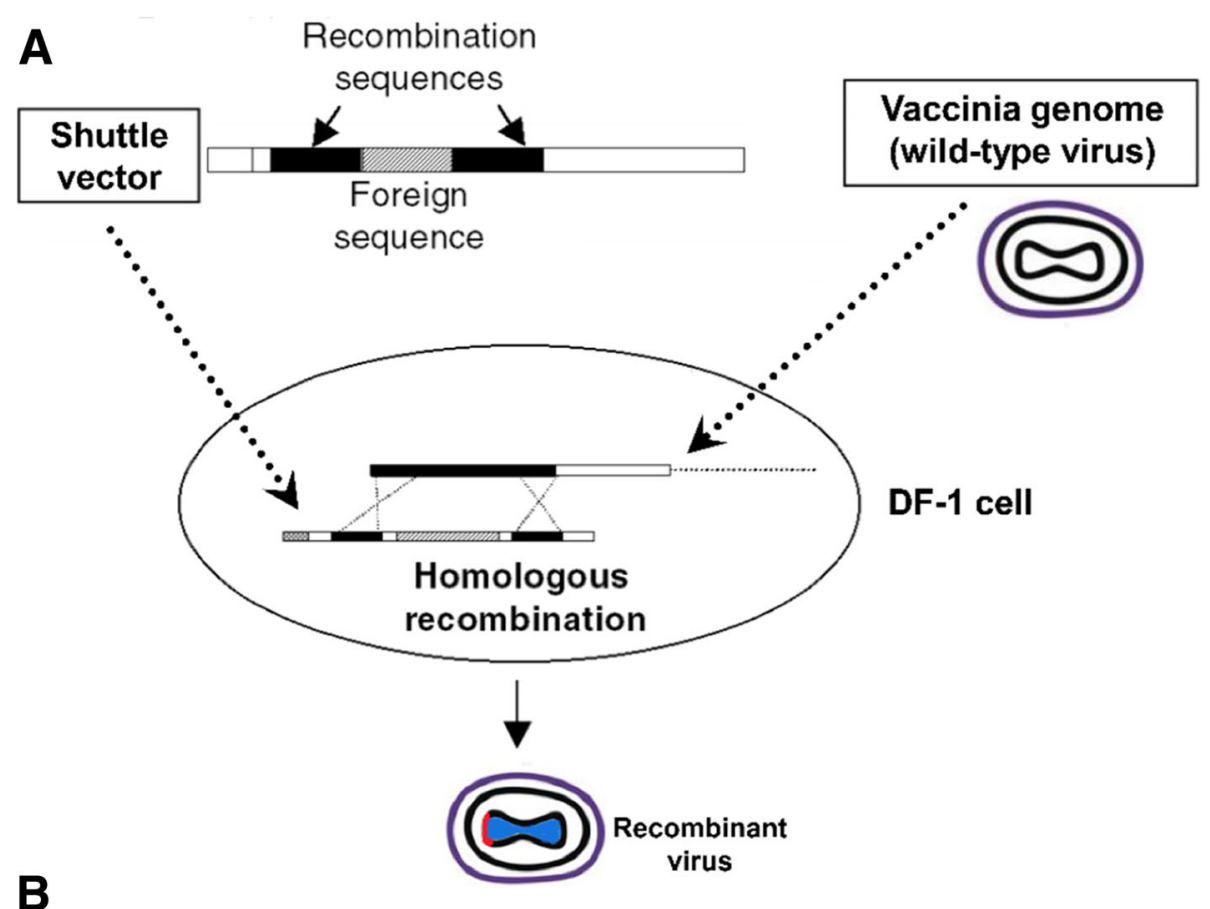

B

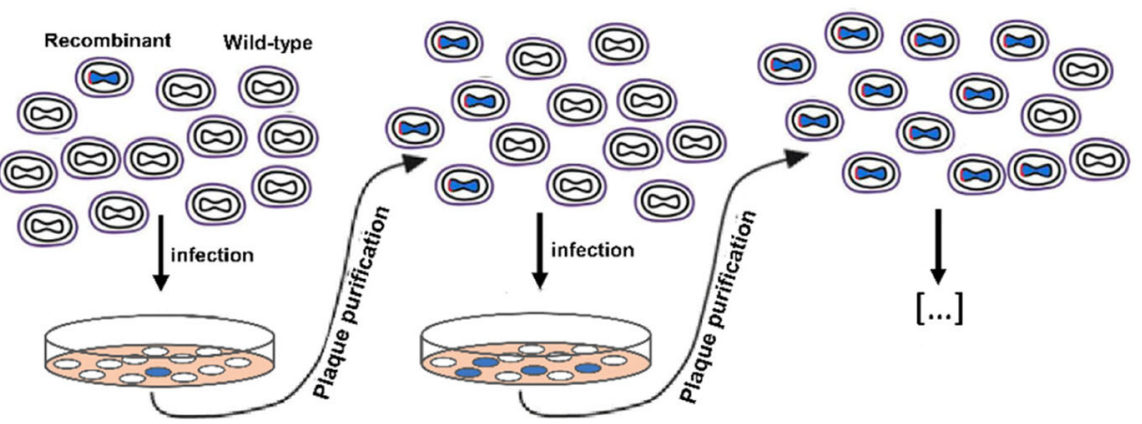

Fig. 1 Schematic overview of traditional marker-based method for generation of recMVA. a Construction of a recombinant vaccinia virus by homologous recombination in DF-1 cells infected with WT MVA. b Plaque purification of recMVA. Adapted from Nagel [13] and Rocha [14] with modifications 


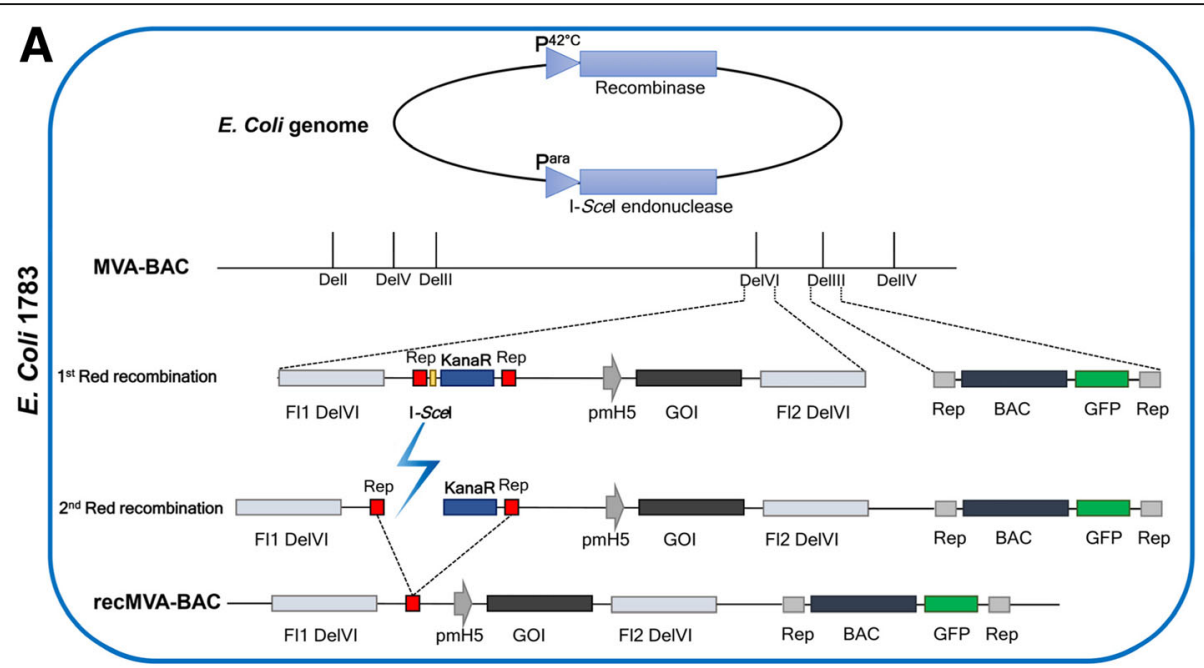

B

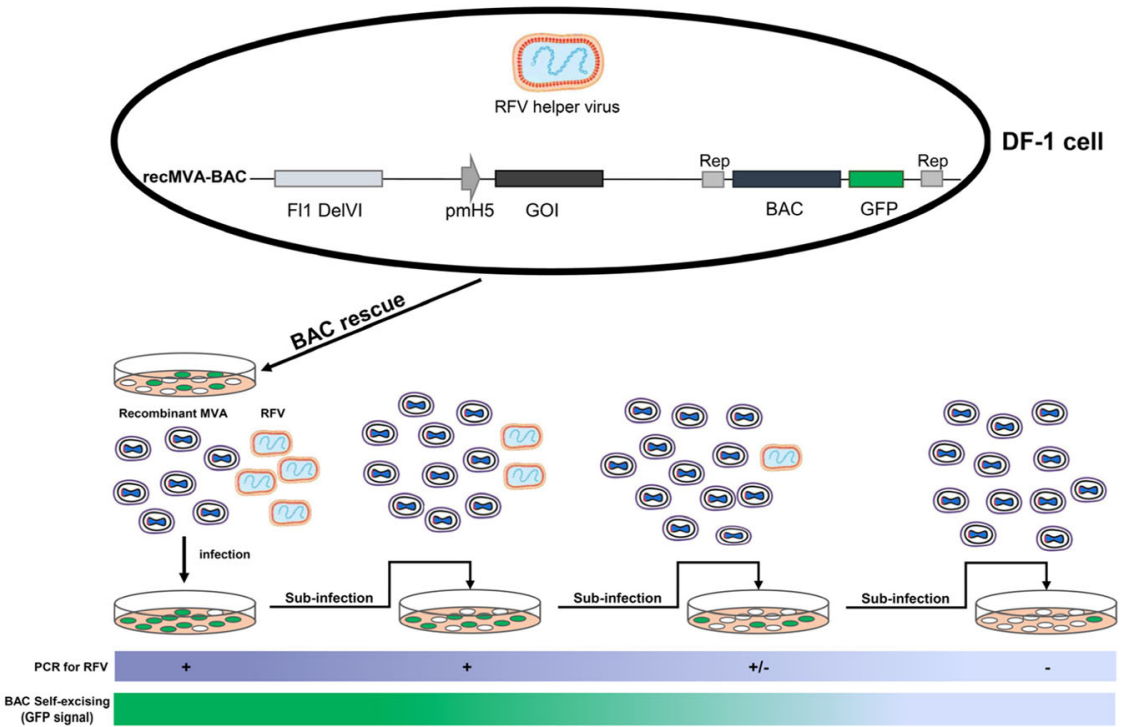

Fig. 2 Schematic overview of MVA-BAC technique for generation of recMVA. a In E. coli GS1783, following induction at $42^{\circ} \mathrm{C}$, the gene of interest together with I-Scel-Kan fragment is inserted into the DeNI site of the MVA-BAC by homologous recombination. Co-integrated Kan cassette (aphAl) is removed by induction of I-Scel production by arabinose followed by second Red recombination $\mathbf{b}$ In DF-1 cells, recMVAs are rescued in present of a helper virus and the BAC backbone including GFP cassette is removed spontaneously upon passaging

recombination. I-SceI endonuclease induction is triggered by arabinose to eliminate Kan resistance gene. Subsequently, to rescue recombinant progeny virus, recMVA-BACs are introduced into DF-1 cells, as presented in Fig. 2b. In this step, a helper virus which provides the initial transcription machinery for triggering recMVA replication, is required. As the BAC construct contains a GFP cassette, successfully infected cells can be monitored by fluorescence microscopy. In a final step, by sub-infection of DF-1 cells with the recMVAs and due to self-excising property of the system, BAC backbone containing GFP cassette is eliminated. The helper virus will also be cleared by passaging steps in
DF-1. These can be confirmed by PCR using specific primers for $g f p$ gene and helper virus genome.

\section{Results}

Generation of recombinant MVA by homologous recombination in DF-1 cells

NoV GII.4 capsid gene was inserted into the BamHI site of the pIIIPH5 Red K1L plasmid (Fig. 3b) to construct a shuttle vector, namely pIIIH5-GII4VP1. After transformation into $E$. coli DH5, insertion was verified by $\mathrm{PCR}$ and restriction enzyme digestion analysis. The integrity of the inserts was confirmed by sequencing. 


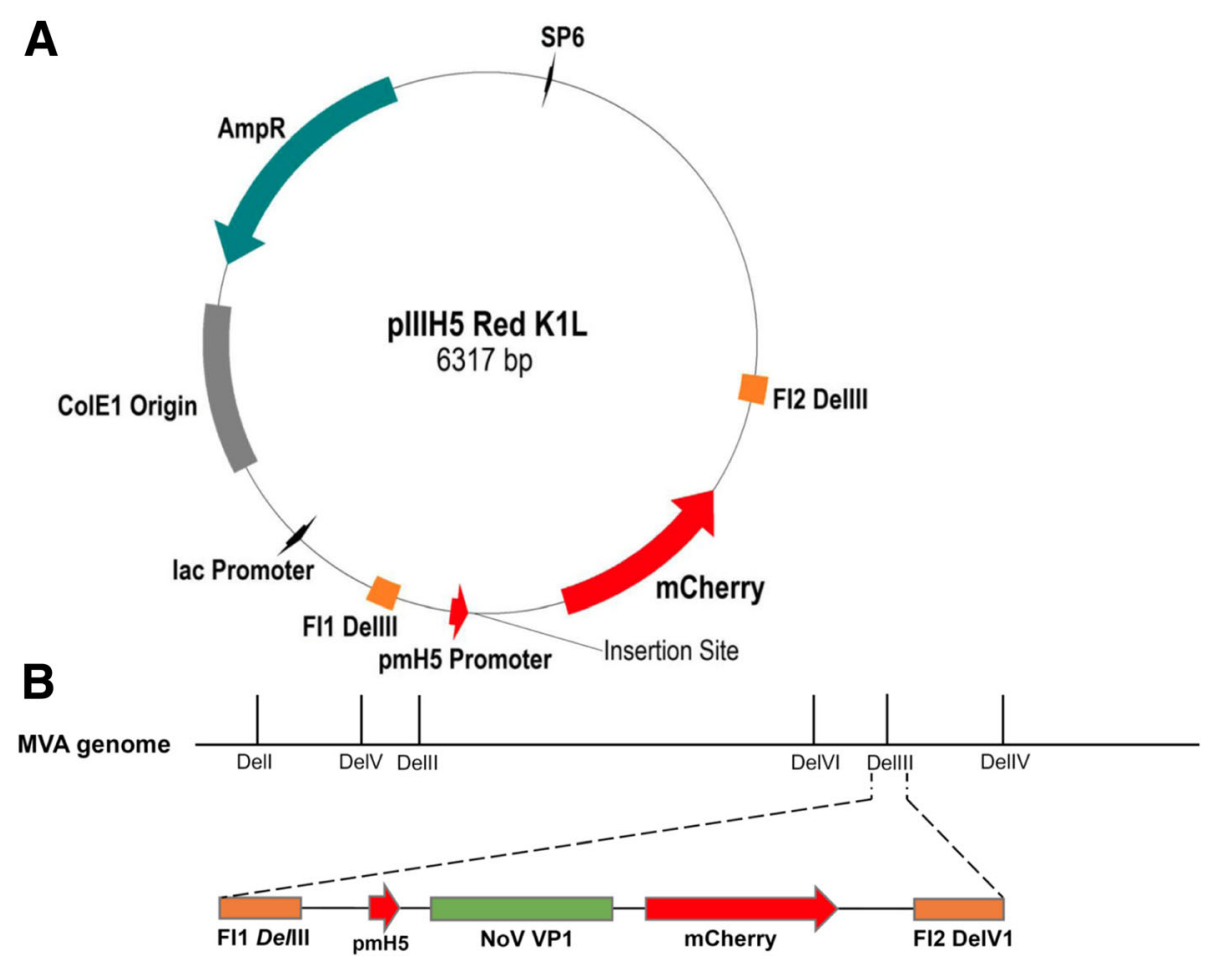

Fig. 3 a Schematic map of shuttle vector plIIH5 Red K1L. b Targeting expression cassette into MVA Dellll region by homologous recombination of adjacent flank sequences FI1 Dellll and FI2 Dellll

The shuttle vector was introduced into DF-1 cells infected with the WT-MVA. Forty-eight hours posttransfection, the cells were checked for expression of mCherry. As shown in Fig. 4a, in the 1st passage, we could detect red plaques producing recMVA. Insertion of NoV capsid gene was confirmed by PCR (Fig. 4b). In each step, the presence of the WT MVA was checked by PCR. After several rounds of plaque purification, WTMVA DNA became undetectable for the recMVA-GII.4 (Fig. 4c).

\section{Generation of recombinant MVA-BAC}

Recombinant shuttle vectors carrying NoV capsid gene were generated by insertion of the GII.4 VP1 gene into the $B g l \mathrm{II}$ and $\mathrm{XbaI}$ sites of the pEPMVAdVI PH5 plasmid downstream of the PmH5 promoter (Fig. 5b). After transformation into E. coli competent cells, correct clones were identified by RE digestion and PCR using gene-specific and DelVI primers. Potential correct clones were checked for integrity of the inserts by sequencing. To generate recombinant MVA-BAC, the transgene cassette along with the Kan cassette, I-SecI sequence and DelVI flanking sequences were amplified from the shuttle vectors and introduced into the GS1783 E. coli competent cells carrying the MVA/BAC. As shown in Fig. 5c, PCR amplification confirmed the insertion of the I-SecI-Kan-pH 5/NoVGII4 fragment into the MVA genome in the BAC plasmid. The KanS-cassette was removed by arabinose induction of endonuclease, I-SceI followed by induction of the second Red recombination at $42{ }^{\circ} \mathrm{C}$ in the GS1783 E. coli cells. Correct clones were confirmed by PCR using specific DelVI primers (Fig. 5d). Restriction fragment analysis using BamHI and EcoRI also confirmed correct insertion of the VP1 capsid gene (Data not shown).

The MVA-BAC-GII4VP1 plasmid was transfected into DF-1 cells after infection of the cells with the helper virus RFV. After 2-3 days of incubation at $37^{\circ} \mathrm{C}$, the cells were harvested and the recombinant viruses were used to infect new DF-1 cells. PCR analysis revealed successful integration of the capsid genes (Fig. 6a). Clearance of the helper virus from the recMVA-producing cells was confirmed, after four rounds of passaging (Fig. 6b). Due to self-excising property of the MVA-BAC, the BAC-GFP cassette was expected to be eliminated from the MVA genome by an inverse-oriented sequence replication in the DelIII of the genome [15]. As shown in Fig. 6d, after five rounds of recMVA passaging we observed a significant reduction in GFP population indicating the loss of BAC cassette from the DelIII locus of the recMVA genome. Successful removal of BAC-GFP marker was assured by PCR using specific primers for the $g f p$ gene, as shown in Fig. 6c. 

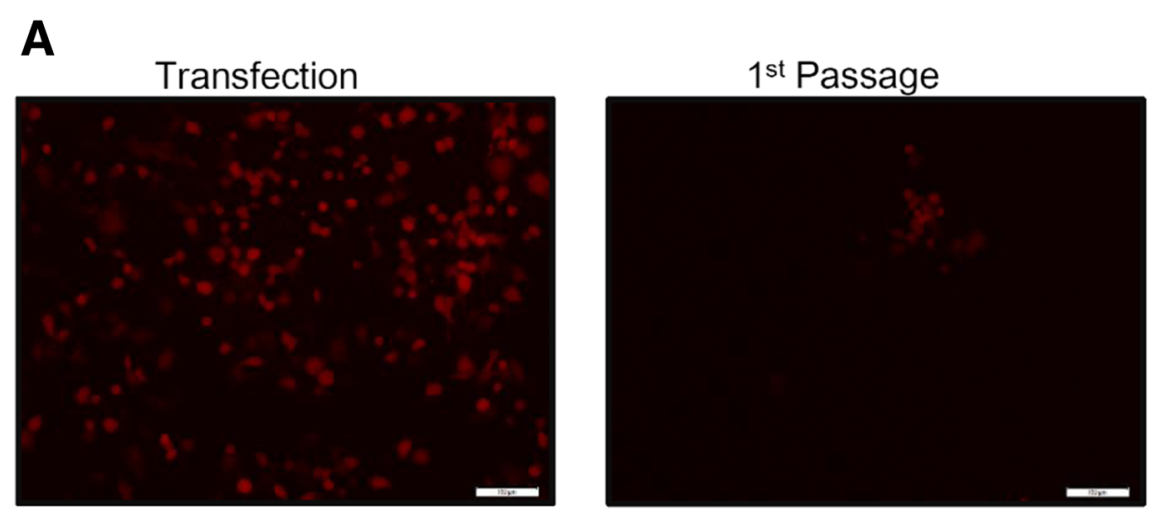

$3^{\text {rd }}$ Passage

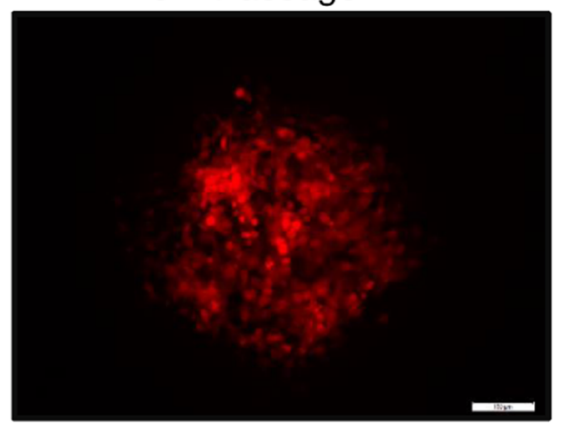

$5^{\text {th }}$ Passage

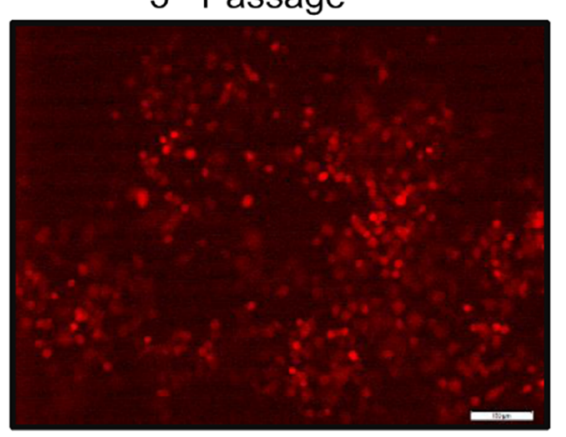

\section{B}
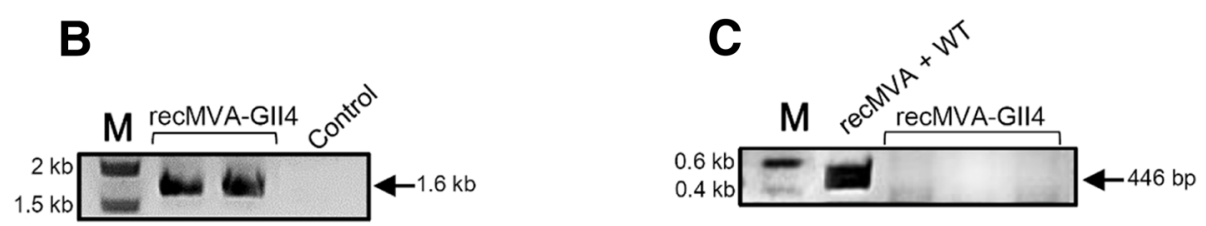

Fig. 4 Generation of recMVA-NoV using marker-based approach. a After transfection of the plIIH5-GII4VP1 into MVA-infected DF-1 cells, mCherryexpressing cells producing recMVAs were picked and plaque purification was carried out. After some rounds of plague picking, a significant increase in the signal was observed. Scale: $100 \mu \mathrm{m}$. b Confirmation of gene insertion into the MVA genome by PCR. After extraction of viral DNA from infected DF-1 cells, insertion of the NoV capsid gene was checked by amplifying the Dellll cassette with gene-specific primers. An amplified fragment of $1.6 \mathrm{~Kb}$ was expected for the Gll.4. DF-1 cells infected with WT-MVA were used as negative control for PCR. c Confirmation of WTMVA clearance. The presence of the WT-MVA was checked by amplifying Dellll cassette where a PCR product of 446 bp was expected

\section{Biochemical characterization of the recMVAs}

Binding assay clearly showed binding of the recMVAs on the cell surface, as shown in Fig. 7a. Propagated recMVAs were purified from cell suspension and after negative staining were visualized by EM where enveloped, brick-shaped MVA virions with a size of approximately $300 \times 250 \mathrm{~nm}$ [16] were detected (Fig. 7b). Successful expression of NoV capsid protein in the recMVA-producing DF-1 cells was confirmed by Western blotting, where we could clearly detect a $58 \mathrm{kDa}$ VP1 band for both constructs (Fig. 7c). A $32 \mathrm{kDa}$ protein band, the P domain size, was also detected. According to a previous study [17], soluble VP1 is susceptible to in vivo proteolytic trypsin cleavage at aa residue 227 , leaving a $32 \mathrm{kDa}$ protein. The cleavage site is thought to be buried in assembled virions, while it becomes exposed when the particles assemble to multimers or unfold. The absence of the $\mathrm{S}$ domain is thought to be due to intercellular degradation $[15,18]$. The bands we assume as $\mathrm{P}$ particles or multimers were not present in the uninfected control. Recombinant GST-tagged GII.4 VP1 protein expressed in E. coli and non-infected cells were used as positive and negative controls, respectively. Plaque-forming units (PFU) were determined for the virus stocks in DF-1 cells infected in duplicates with the respective virus in 10-fold serial dilutions. Virus titers of $4.1 \times 10^{8}$ - and $1.7 \times 10^{9} \mathrm{PFU} / \mathrm{ml}$ were measured for the marker-based-and the BAC-based systems, respectively.

\section{Discussion}

The highly attenuated vaccinia virus, MVA, has been established as a safe and potent viral vector for the development of recombinant vaccines [7, 19-21]. MVAbased vaccines have been shown to induce a robust $\mathrm{T}$ 

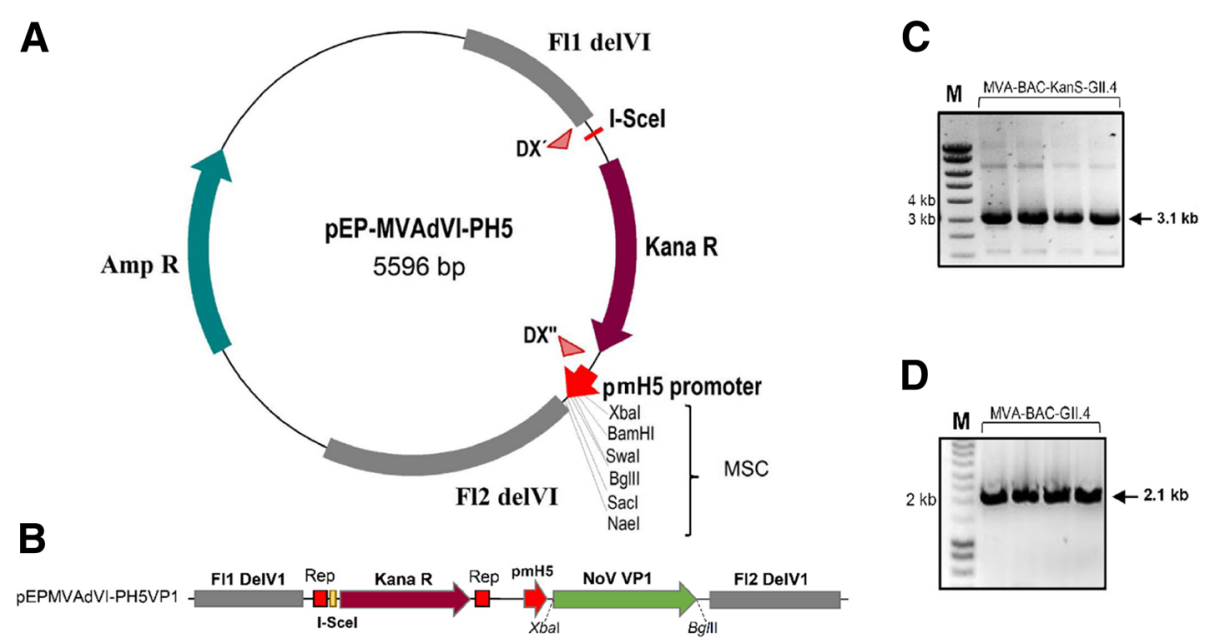

Fig. 5 Generation of recMVA-NoV BAC plasmid. a Schematic map of the shuttle vector pEP-MVAdVI-PH5. $\mathbf{b}$ Linear map of insertion region in the recombinant pEPMVAdVI-PH5VP1. The recombinant plasmid was constructed by insertion of the NoV GIl.4 capsid gene VP1 into the shuttle vector downstream of the viral promoter PmH5. c Confirmation of first Red recombination. After first recombination in E. coli 1783, insertion of the I-Secl-Kan-pH 5NP1 fragment into MVA-BAC genome was confirmed by PCR (expected size: $3.1 \mathrm{~kb}$ ). $\mathbf{d}$ Confirmation of resolution of cointegrated Kan cassette by PCR using DeNl-specific primers (expected size: $2.1 \mathrm{~kb}$ )

cell response to the transgene [16, 22, 23]. A short burst of antigen expression following immunization makes it also suited for B cell boosting [24]. In the present study, we generated recMVA vectors encoding norovirus GII.4 capsid protein to be used as B- and T- cell booster in a prime-boost vaccination regime. In this work, beside the available marker-based approach, a BAC-based system was applied for generation of recombinant MVA vectors. The marker-based approach relies upon homologous recombination with a shuttle vector in virus-infected

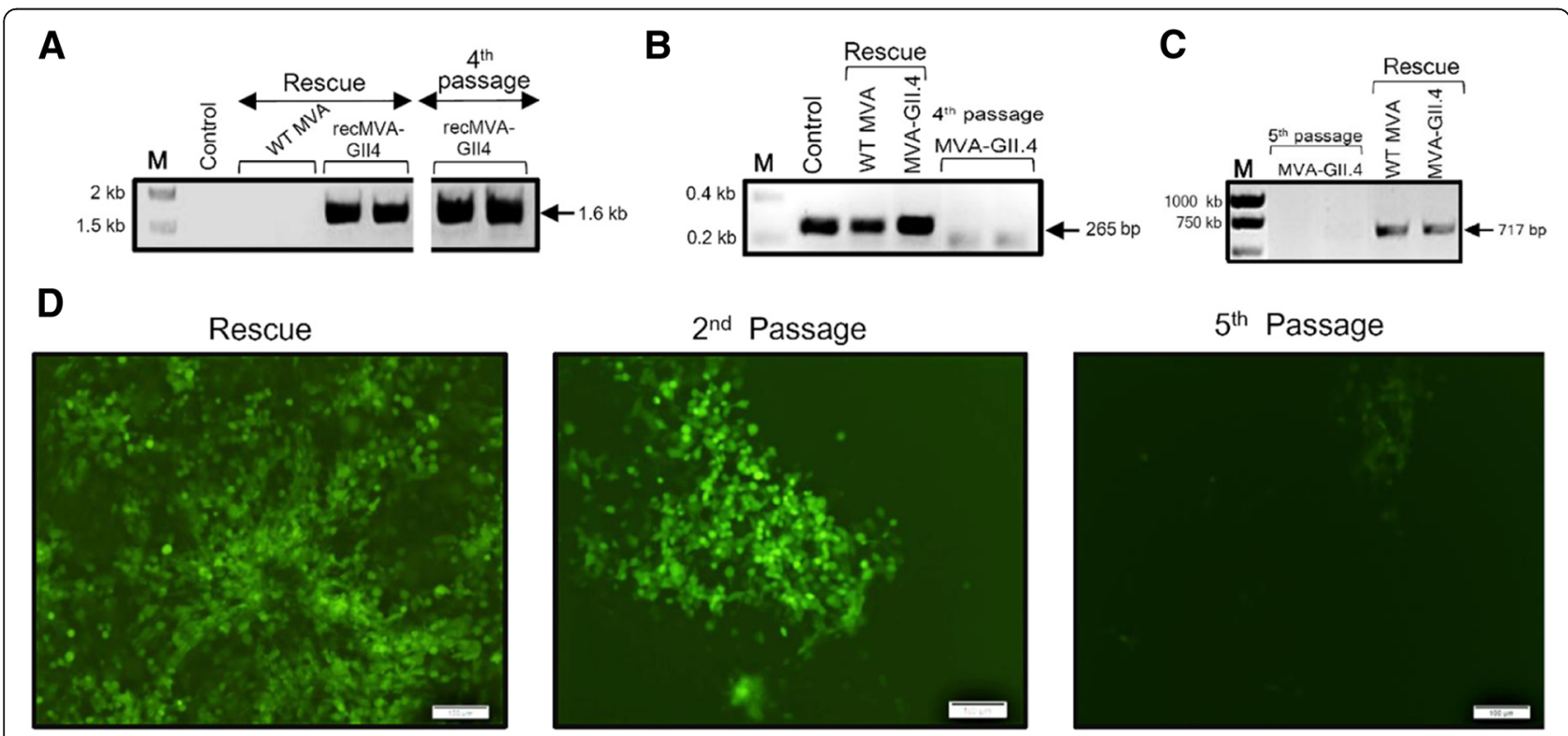

Fig. 6 Rescue of recMVA-NoV from recBAC plasmid in DF-1 cells. a Confirmation of gene insertion into the MVA genome by PCR. Insertion of the capsid gene was checked by amplifying the DeNI cassette using DeNI primers for the WT (expected size: 498 bp) and gene-specific primers for the recMVA-BAC-GIl.4 (expected size: $1.6 \mathrm{~kb}$ ). Uninfected DF-1 cells were used as negative control. b Confirmation of RFV clearance by PCR using RFV-specific primers with an expected size of $265 \mathrm{bp}$. RFV-related band was detected in the first rescue sample; it was not detectable from the 4th passage. Purified RFV was used as positive control. $\mathbf{c}$ and $\mathbf{d}$ BAC self-excision: After transfection of the shuttle vector into RFV-infected DF-1 cells, the BAC backbone and the GFP cassette were spontaneously lost by passaging: (C) Confirmation of removal of BAC backbone by PCR using specific primers for gfp gene. (D) significant reduction in GFP population indicating the loss of BAC cassette from the recMVA genome. Scale: $100 \mu \mathrm{m}$ 


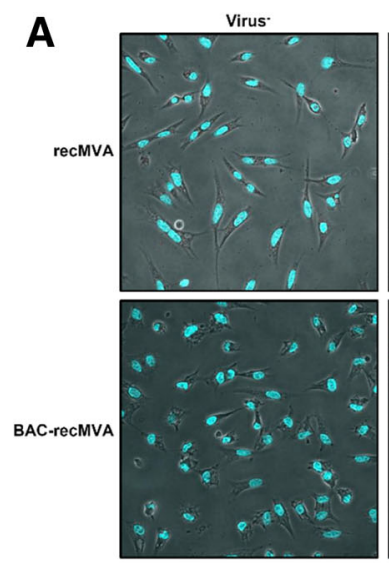

C

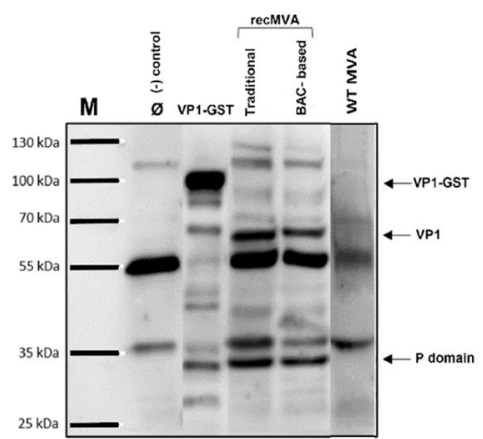

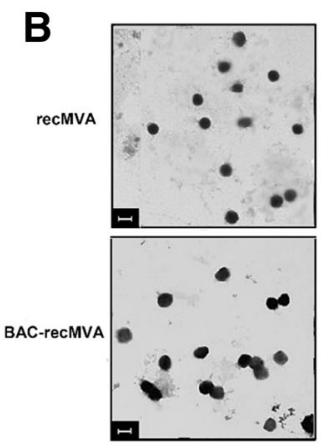
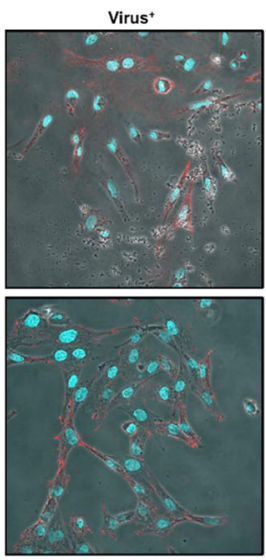

D

Fig. 7 Biochemical characterization of recMVAs. a Binding assay: Control cells are depicted on the left side. Virus binding to DF-1 cells was visualized by staining with anti-Vaccinia virus antibody. Top: recMVAs derived by the marker-based method; Bottom: BAC-recMVAs. b Electron microscopy with negative staining. Scale: $300 \mathrm{~nm}$. c Expression of NoV VP1 in DF-1 cells infected with recMVAs. Immunoblotting was carried out using human serum from Gll-infected patients as primary- and anti-human IgG as secondary antibody. VP1 protein (58 kDa) was detected in both recMVAs. Non-infected- and WT-MVA-infected cells were used as negative controls. Recombinantly expressed GST-tagged Gll.4VP1 was used as positive control. d Schematic workflow for recMVA generation using traditional approach (left) and MVA-BAC system (right)

cells. We used pIIIH5 Red K1L as shuttle vector where the NoV GII.4 capsid gene was inserted downstream of the modified $\mathrm{H} 5$ promoter PmH5 which contains both native early and late vaccinia promoter regions [25]. PmH5 is a strong vaccinia virus promoter that overexpresses the respective antigens in a high dose, during the early and late phase of virus infection [12]. It has been shown that antigen driven by PmH5 elicited dramatically a higher immunogenicity than those by other vaccinia virus promoters $[26,27]$. In addition, $\mathrm{mH} 5$ promoter improves stability of insert genes during extended passages when compared with some other strong promoters such as pSyn I or II [12]. The transgene was designed so that it can recombine into the DelIII site of the MVA genome by homologous flanks adjacent to the gene of interest. Forty-eight hours after introducing the shuttle vectors into DF-1 cells, recMVA-producer cells were recognized by mCherry expression. In early passages, plaque picking was tedious, as only a few cells were infected and a high number of plaques had to be isolated for the second round of purification. After several rounds of plaque purification from infected cells with highly diluted virustiters we managed to eliminate wild type MVA. The reporter gene mCherry is removed by spontaneous homologous recombination of two flanking sequences. Therefore, after some rounds of plaque purification we expected a decrease in fluorescence signal with an increase in the recMVA yield. Eliminating the reporter gene is particularly important when the potential vaccine is required for clinical applications. The production of this markerless construct brought up another drawback as MVA does not form morphologically distinct plaques in DF-1 cells. Hence, picking of mCherry-negative plaques issued another challenge. We could not eliminate the mCherry reporter marker after 10 rounds of passaging (data not shown). Even though the marker-based generation of MVA recombinants is robust, purification of the rare spontaneous recombinants is time-consuming and cumbersome.

In the BAC-based system, a self-excising variant of MVA-BAC was applied in which the BAC cassette harboring the selection marker is spontaneously removed 
from derived recombinant viruses. The major advantage compared to the marker-based attempt is the utilization of $E$. coli as a production platform. In this way, positive clones harboring the expression cassette can easily be identified by their Kanamycin resistance. In contrast, the only way to determine positive clones in the markerbased system is mCherry expression in infected DF-1 cells. Hence, the MVA-BAC system guarantees the successful incorporation of the gene of interest at a stage much ahead of the actual production of virions in DF-1 cells. GFP-expression facilitated evaluation of correct transfection of DF-1 cells significantly and, importantly, the cassette was lost after few rounds of sub-passaging. Compared to the marker-based approach, the elimination of the marker gene scar was found to be vastly superior in the BAC-system. In contrast to the markerbased methods, where several rounds of plaque purification are required for elimination of the WT virus from the culture, in MVA-BAC approach RFV, providing initial transcriptional products for virus replication, was eliminated very fast, after two rounds of passaging. RFV infection in DF-1 cells is abortive as the virus is unable to replicate in these cells.

After successful production of recMVAs, stable integration of the capsid gene into the genome was confirmed. Successful expression of the capsid protein was then confirmed by visible VP1 band in Western blot for the recombinants. With electron microscopy, we could see the expected round $300 \times 250 \mathrm{~nm}$ viral particles [16] We also visualized recMVAs by a binding assay. Attachment of the recombinant MVA particles on the DF-1 cell surface was clearly visible; even though our subjective impression suggested a higher binding rate for the recMVA-BACs.

Not only high virus titer was measured with recMVABACs, but also infection of new DF-1 cells was more efficient than with the recMVAs obtained by the marker-based method. We therefore hypothesize that the MVA-BAC platform provides more functional viral particles. This might be due to the location of transgene insertion. The marker-based approach uses DelIII, whereas MVA-BAC aims for DelVI. DelIII has occurred in earlier passaging stages during MVA generation whereas DelVI has occurred 190 passages later. Interestingly, these deletion sites are in close proximity within the MVA genome. It still is not known what effects the deletion sites have on attenuation [28, 29]. However, these factors may contribute to the anticipated different protein expression levels as well as infection- and amplification properties of the virus.

\section{Conclusions}

In this study, we successfully constructed recombinant MVA expressing norovirus capsid protein of GII.4 genotype by two different approaches. We conclude that the MVA-BAC system quickly produces high amounts of functional recombinant viral particles. The generation of the expression cassette, manipulate the MVA genome and removal of the selective marker resistance gene is done in E. coli, which simplifies handling and generation of recombinant MVAs.

Although MVA is known to be a potent boosting vector, it has limitations for priming immunostimulatory responses [20]. MVA can induce $\mathrm{CD}^{+}$and accompanying $\mathrm{CD} 4^{+} \mathrm{T}$ cell response and a short burst of antigen expression also boosts B cells $[16,19,21]$. Therefore we suggest using recMVAs as booster for B- and T- cell responses in combination with protein-, DNA- and/or mRNA vaccines.

\section{Methods}

Plasmids, bacterial strains, cells and viruses

DF-1 chicken fibroblast cells were used for MVA replication. Wild-type MVA [30] was obtained from the Institute of Infectious Diseases and Zoonosis, University of Munich LMU. MVA-BAC plasmid and helper Rabbit Fibroma Virus (RFV) was obtained from the Institute of Virology, Universitätklinikum Düsseldorf. E. coli GS1783 was used for generation of recMVA-BAC plasmid. Cloning plasmid pEX-K4-NoVGII.4 was used as a template for amplification of the NoV capsid gene. The pIIIPH5-RedK1L plasmid (Fig. 3a) was used as a shuttle vector for generation of recMVA by the marker-based approach. It contains an expression unit under control of a PmH5 promoter [12]. Homologous recombination with the non-essential DelIII of MVA is directed by two stretches of genomic MVA sequences flanking the foreign gene and the mCherry cassette. The pEP-MVAdVI-PH5 plasmid containing two homologous sequences to the DelVI of the MVA genome was used as a shuttle vector for generation of recMVABAC. It contains MVA DNA homologous flanking sequences (FL1/2) adjusted to the MVA deletion VI (DelVI) region. The plasmid encodes for the ampicillin resistance gene as well as for the kanamycin resistance gene aphAI. There is an 18-bp recognition site of the endonuclease I-SceI upstream the Kan cassette. I-SceI generates a double-strand break at the recognition site without cleavage of human, bacterial or viral DNA [31]. Adjacent to the I-SceI sequence and the Kan cassette, the 51 bp homologous sequences DX' and DX' ensure the elimination of the marker gene by homologous recombination.

\section{Generation of recMVA in MVA-infected DF-1 cells Construction of MVA shuttle vector}

The full length of NoV GII.4 capsid gene was amplified from the pEX-K4-GII.4 plasmid using a pair of 
Table 1 List of primers used in this study

\begin{tabular}{|c|c|c|}
\hline Primer & Sequence $5^{\prime} \rightarrow 3^{\prime}$ & Description \\
\hline Kan control & $\begin{array}{l}\text { F: CGTACTCCTGATGATGCATG } \\
\text { R: ATTCGTGATTGCGCCTGAGC }\end{array}$ & $\begin{array}{l}\text { For control PCR/sequencing after } \\
\text { first recombination into MVA-BAC }\end{array}$ \\
\hline MVA De/lll & $\begin{array}{l}\text { F: GATGAGTGTAGATGCTGTTATTITG } \\
\text { R: GCAGCTAAAAGAATAATGGAATTG }\end{array}$ & $\begin{array}{l}\text { To check the presence of } \mathrm{WT} \text { and } \\
\text { gene insertion after plaque picking }\end{array}$ \\
\hline MVA DeNl & $\begin{array}{l}\text { F: CTCCGCATCTAGTTGATATTCCAACCTCTT } \\
\text { R: CCTGGACATTTAGTTTGAGTGTTCCTGAAT }\end{array}$ & $\begin{array}{l}\text { For first homologous recombination } \\
\text { into the MVA-BAC by En-passant }\end{array}$ \\
\hline pEPH5-GII4 & $\begin{array}{l}\text { F: CATAAATAAGGTTGACTCTAGAGCCACCATGAAGATGGCCTC } \\
\text { R: ACGTAGAGCTCTTAAGGAATTCTAATACGGCTCGTCTTCTACCT }\end{array}$ & $\begin{array}{l}\text { For cloning of NoV Gll.4 into } \\
\text { pEP-MVAdVI-PH5 plasmid }\end{array}$ \\
\hline pll|H5-GIl.4 & $\begin{array}{l}\text { F: TAAGGTTGACTCTAGAGCTAGCGCCACCATGAAGATGGCCTC } \\
\text { R: CGGCCGCGTTTAAACCTCGAGTTATACGGCTCGTCTTCTACCT }\end{array}$ & $\begin{array}{l}\text { For cloning of NoV Gll.4 into } \\
\text { the plllH5 shuttle vector }\end{array}$ \\
\hline RFV & $\begin{array}{l}\text { F: AAAGATGCGTACATTGGACCC } \\
\text { R: GTTCGAGACTAGAAAAGCGCC }\end{array}$ & $\begin{array}{l}\text { To check the presence of helper virus RFV } \\
\text { in the transfect cells with recMVA-BAC }\end{array}$ \\
\hline
\end{tabular}

specific primers listed in Table 1, and inserted into the BamHI site of the shuttle vector pIIIH5-RedK1L downstream of the PmH5 promoter using In-Fusion cloning kit (Takara Bio, USA). After transformation into Top10 E. coli competent cells, positive clones were screened on LB-Amp $(100 \mu \mathrm{g} / \mathrm{ml})$ plates. Recombinant plasmids were checked for gene insertion by restriction enzyme (RE) digestion. Integrity and correct orientation of the insert were verified by double-stranded sequencing. The constructs were purified in large-scale using the Plasmid Plus Midi Kit (Qiagen, Germany).

\section{Generation of recMVA by homologous recombination}

At confluency of approximately 90\%, DF-1 cells were infected with WT-MVA at MOI 0.05 for $1.5 \mathrm{~h}$ at $37^{\circ} \mathrm{C}$. The cells were then transfected with the pIIIH5-RedK1L-GII.4VP1 plasmid using Lipofecta$\min ^{\odot} 2000$ transfection reagent (Thermo Scientific, USA) as described by Moeini et al. [32]. Forty-eighthour post-transfection, the cells were harvested by scraping and centrifugation at $4000 \mathrm{rpm}, 4{ }^{\circ} \mathrm{C}$ for 10 min. The cells were resuspended in $1 \mathrm{ml}$ media and viral particles were released from the cells by 3 times freeze-thawing. Cell debris was pelleted at 14, $000 \mathrm{rpm}$ for $10 \mathrm{~min}$, and $200 \mu \mathrm{l}$ of the virus-containing supernatant was used to infect new DF-1 cells in 6 -well plates in a serial dilution $\left(10^{-1}-10^{-6}\right)$. Two days post-infection, the cells were checked under fluorescence microscope for the expression of the reporter gene, mCherry. Red plaques were picked and used to infect new DF-1 cells for further purification of the mCherry-expressing plaques. Plaque picking was carried out for several rounds to get rid of the WT-MVA. In each step of plaque purification, the cells were checked for the presence of the WT-MVA by PCR using DelIII-specific primers listed in Table 1.

\section{Generation of recMVA by BAC-based system} Construction of MVA shuttle vector

Norovirus GII.4 capsid gene was amplified from the pEX-K4GII.4 plasmid using a pair of specific primers listed in Table 1 and inserted into the $\mathrm{XbaI}$ and BglII sites of the shuttle vector pEP-MVAdVI-pH 5 using InFusion cloning kit. After transformation into E. coli competent cells, positive clones were screened on LB-Amp agar; plasmids were extracted and subsequently gene insertion was confirmed by PCR and RE digestion. Finally, the integrity of the gene insert was confirmed by sequencing.

\section{Insertion of the VP1 gene into the MVA-BAC genome}

E. coli GS1783 carrying MVA-BAC (GS1783/MVA) was grown overnight at $32{ }^{\circ} \mathrm{C}$ in LB broth supplemented with $30 \mu \mathrm{g} / \mathrm{ml}$ chloramphenicol (Cam); electrocompetent cells were prepared as previously described [33]. The GII4 VP1 gene along with the KanS fragment (including Kan cassette and I-SceI restriction site) was amplified from the pEP-MVAdVI-pH 5-GII4VP1 plasmid using MVA DelVI primers listed in Table 1 . The PCR products were treated with $D p n I$, purified and introduced into the electrocompetent E. coli GS1783/MVA cells for the first Red recombination. Briefly, in a pre-chilled electroporation cuvette, 100 ng of the PCR products was added to $50 \mu$ l of the electrocompetent cells and electro-transformation was carried out at $15 \mathrm{kV} / \mathrm{cm}, 25 \mu \mathrm{F}, 200 \Omega$ using the Gene Pulser Xcell Electroporation device (Bio-Rad, USA). The cells were immediately mixed with $1 \mathrm{ml} \mathrm{LB}$ broth followed by $1 \mathrm{~h}$ incubation at $32{ }^{\circ} \mathrm{C}$. Positive clones were screened on LB/ cam agar after $24 \mathrm{~h}$ incubation at $32^{\circ} \mathrm{C}$. Recombinant MVA-BAC plasmids were extracted using the GeneJet Plasmid Miniprep Kit (Thermo Scientific, USA) and gene insertion was confirmed by PCR using the DelVI primers.

To remove the co-integrated Kan cassette from the inserts, in $1 \mathrm{ml}$ LB-Cam broth medium, E. coli cells carrying recombinant MVA-BAC were inoculated at $32{ }^{\circ} \mathrm{C}$ and 220 rpm for $2 \mathrm{~h}$ until the culture became cloudy. Thereafter, to 
induce the production of I-SceI, $1 \mathrm{ml}$ pre-warmed LB-Cam medium $+2 \%$ arabinose was added to the culture followed by $1 \mathrm{~h}$ incubation at $32^{\circ} \mathrm{C}$ and $220 \mathrm{rpm}$. The cells were then heat-shocked at $42^{\circ} \mathrm{C}$ in a shaker water bath for $30 \mathrm{~min}$ to induce Red recombinase. After $2-3 \mathrm{~h}$ incubation at $32^{\circ} \mathrm{C}$ in a shaker incubator, the cells were screened on a LB-Cam agar plate supplemented with $1 \%$ arabinose. After 2 days of incubation at $32{ }^{\circ} \mathrm{C}$, positive clones were tested for the resolution of the co-integrated cassette by PCR.

\section{MVA BAC-rescue in DF-1 cells}

In 6-well plates, at confluency of $80-90 \%$, DF-1 cells were transfected with the recMVA- BAC GII4VP1 plasmid using Lipofectamin 2000 transfection reagent. For reactivation of MVA, after $3 \mathrm{~h}$ incubation at $37^{\circ} \mathrm{C}$, the cells were infected with the helper virus RFV at MOI 1 with gentle rotation [34]. One hour later, the wells were filled up with RPMI medium supplemented with $10 \%$ FCS. After $24-48 \mathrm{~h}$ incubation at $37^{\circ} \mathrm{C}$, the cells were checked for the expression of GFP with the Fluorescence Microscope CKK41 (Olympus, Germany).

After successful rescue of viral progeny from the BAC, to remove the helper virus, the cells were harvested by scraping and spun at $4000 \mathrm{rpm}$ and $4{ }^{\circ} \mathrm{C}$ for $10 \mathrm{~min}$. The cell pellet was resuspended in $1 \mathrm{ml}$ DMEM GlutaMAXX (Gibco/Thermo Scientific, USA) and viral particles were released from the cells by 3 times freeze-thawing. Cell debris was precipitated by $10 \mathrm{~min}$ centrifugation at 14 , $000 \mathrm{rpm}$. Two-hundred $\mu \mathrm{l}$ of the virus-containing supernatant in serial dilutions $\left(10^{-1}-10^{-6}\right)$ was used to infect new DF-1 cells at confluency of $70-80 \%$, in a 6 -well plate. Three days later, cells from the most infected wells were harvested and blind passages were repeated until RFV was eradicated. This was tested by PCR using the RFV-specific primers listed in Table 1 . Passages were continued to get rid of the plasmid backbone and GFP cassette from the viral genome. Finally, in positive recMVA-producing cells, the integrity of the gene inserts was checked by sequencing of the DelVI PCR product.

\section{Virus stock preparation by ultracentrifugation}

DF-1 cells were seeded in T-75 flasks and then at confluency of $80-90 \%$ were infected with the recMVA viruses. Two days post-infection, the cells were harvested by scrapping; washed with $1 \times \mathrm{PBS}$ and then resuspended in $10 \mathrm{mM}$ Tris $\mathrm{pH} 9$. After 3 times freezethawing, the samples were sonicated 3 times on ice, each for $30 \mathrm{~s}$, at $100 \%$ power. The cell debris was sedimented at $4000 \times g, 4{ }^{\circ} \mathrm{C}$ for $5 \mathrm{~min}$. In UltraClear tubes (Bechman Coulter, Germany), the supernatant containing viral particles was added to $36 \%$ sucrose and viral particles were pelleted with ultracentrifugation at $13,500 \mathrm{rpm}$ and $4{ }^{\circ} \mathrm{C}$ for $1.5 \mathrm{~h}$. Virus pellets were air-dried and resuspended in $200 \mu \mathrm{l} 10 \mathrm{mM}$ Tris pH 9 for further analysis.

\section{Electron microscopy}

Negative staining was carried out to visualize recMVA particles by electron microscopy (EM). For this, $5 \mu \mathrm{l}$ of the purified virus suspension was put on a S162 flying copper grid. After $5 \mathrm{~min}$, the copper grid was washed with $5 \mu \mathrm{l}$ water and then were stained with $0.5 \%$ uranyl acetate in water for $20 \mathrm{~s}$. The fluid film was removed with a paper and the samples were then analyzed by EM.

\section{Immunofluorescence staining for binding assay}

In 12-well plates, UV-sterilized cover slips were coated with 1:20 diluted L-lysine in PBS at $37^{\circ} \mathrm{C}$ for $1 \mathrm{~h}$. After washing with $1 \times \mathrm{PBS}$, DF- 1 cells were seeded on the coated cover slips. At confluence of $70-80 \%$, the culture medium was removed; the cells were washed twice with ice-cold PBS and then incubated with virus supernatant at $4{ }^{\circ} \mathrm{C}$ for $2-3 \mathrm{~h}$. The cells were then washed $(3 \mathrm{x})$ carefully with PBS; fixed with $4 \%$ PFA for 20 min and then incubated for $30 \mathrm{~min}$ at $37^{\circ} \mathrm{C}$ with anti-Vaccinia virus primary antibody (Acris Antibodies, Germany) diluted in PBS $+10 \%$ serum. Slides are washed twice, each for 5 min, with $1 \times$ PBS before adding goat Alexa Fluor 594conjugated anti-rabbit IgG $(\mathrm{H}+\mathrm{L})$ secondary antibody in PBS $+10 \%$ serum. After $30 \mathrm{~min}$ incubation at $37^{\circ} \mathrm{C}$ in a dark chamber, the cover slips were washed $(\times 2$, each for $5 \mathrm{~min}$ ) and then transferred upside down to a glass slide with DAPI mounting solution. The next day, the cells were analyzed with the Confocal Microscope Fluoview FV10 (Olympus, Germany).

\section{Virus titration - plaque forming units}

Virus titer was measured for three virus preparations. Briefly, ten-fold serial dilutions $\left(10^{-4}-10^{-9}\right)$ of virus suspension in $2 \%$ FCS medium were plated out in duplicates on confluent DF-1 monolayers in 6-well plates. After $2 \mathrm{~h}$ infection at $37^{\circ} \mathrm{C}$, the inoculum was removed and the cells were washed with $1 \times$ PBS followed by 2 days incubation at $37^{\circ} \mathrm{C}$ in $2 \%$ FCS medium. After that, the medium was removed and the cells were fixed with a 1:1 mixture of ice-cold $\left(-20^{\circ} \mathrm{C}\right)$ acetone:methanol for $5 \mathrm{~min}$ at RT. The cells were then blocked for $1 \mathrm{~h}$ at RT with PBS + 3\% FCS and were incubated with rabbit antiVaccinia virus primary antibody (Acris Antibodies, Germany), diluted 1:2000 in PBS + 3\% FCS, for $1 \mathrm{~h}$ at RT. After washing $(\times 3)$ with PBS $+3 \%$ FCS, the cells were incubated with peroxidase-conjugated anti-rabbit IgG (1:5000 in PBS + 3\% FCS) for $1 \mathrm{~h}$ at RT with gentle rocking. The cells were washed $(\times 3)$ and then incubated with the True-Blue Peroxidase Substrate for about 10 min. Stained plaques were counted and virus titer was calculated in $\mathrm{PFU} / \mathrm{ml}$ by multiplying the mean plaque number (big, clearly blue plaques, not including satellite plaques) by the magnitude of dilution. 


\section{Abbreviations}

BAC: Bacterial Artificial Chromosome; CEF: Chicken Embryo Fibroblast; Del: Deletion; GFP: Green Fluorescence Protein; Kan: Kanamycin; MVA: Modified Vaccinia Virus Ankara; NoV: Norovirus; PCR: Polymerase Chain Reaction; PFU: Plaque Forming Unite; recMVA: recombinant MVA; RFP: Rabbit Fibroma Virus; WT: Wild Type

\section{Acknowledgements}

We thank Prof. Gerd Sutter and Michael Lehmann for providing wild-type MVA. We also thank Martin Kächele for helping to produce and biochemically characterize recombinant MVAs.

\section{Authors' contributions}

FK carried out the experiment. ID and UP helped supervise the project and contributed to the final version of the manuscript. $\mathrm{HM}$ and $\mathrm{DH}$ conceived and planned the experiments and supervised the project. All authors read and approved the final manuscript.

\section{Funding}

The $\mathrm{DH}$ received funding from the German Center for Infection Research (http://www.dzif.de/en/); grant number TTU06.804. ID received funding from the German research Foundation (http://www.dfg.de/en/); grant number GRK1449. The funders had no role in study design, data collection and analysis, or preparation of the manuscript.

\section{Availability of data and materials}

All data are fully available without restriction.

\section{Ethics approval and consent to participate}

Not applicable

\section{Consent for publication}

Not applicable

\section{Competing interests}

The authors declare that they have no competing interests.

\section{Author details}

${ }^{1}$ Institute of Virology, Faculty of Medicine, Technische Universität München Munich, Germany. ${ }^{2}$ Institute for Virology, Universitätklinikum Düsseldorf, Heinrich Heine Universität, Düsseldorf, Germany.

Received: 5 April 2019 Accepted: 5 August 2019

\section{Published online: 09 August 2019}

\section{References}

1. Hasing ME, Lee BE, Preiksaitis JK, Tellier R, Honish L, et al. Emergence of a new norovirus GIl. 4 variant and changes in the historical biennial pattern of norovirus outbreak activity in Alberta, Canada, from 2008 to 2013. J Clin Microbiol. 2013:51:2204-11.

2. Zakikhany K, Allen DJ, Brown D, Iturriza-Gómara M. Molecular evolution of Gll-4 norovirus strains. PLoS One. 2012;7:e41625.

3. Boon D, Mahar JE, Abente EJ, Kirkwood CD, Purcell RH, et al. Comparative evolution of GII. 3 and GII. 4 norovirus over a 31-year period. J Virol. 2011:85:8656-66.

4. Thorne LG, Goodfellow IG. Norovirus gene expression and replication. J Gen Virol. 2014:95:278-91.

5. Tan $M$, Jiang $X$. Norovirus $P$ particle: a subviral nanoparticle for vaccine development against norovirus, rotavirus and influenza virus. Nanomedicine. 2012;7:889-97.

6. Sutter G, Staib C. Vaccinia vectors as candidate vaccines: the development of modified vaccinia virus Ankara for antigen delivery. Current Drug TargetsInfectious Disorders. 2003;3:263-71.

7. Drexler I, Staib C, Sutter G. Modified vaccinia virus Ankara as antigen delivery system: how can we best use its potential? Curr Opin Biotechnol. 2004:15:506-12.

8. Kreijtz J, Suezer Y, de Mutsert G, van den Brand J, van Amerongen G, et al Preclinical evaluation of a modified vaccinia virus Ankara (MVA)-based vaccine against influenza a/H5N1 viruses. Vaccine. 2009;27:6296-9.

9. Leung-Theung-Long S, Gouanvic M, Coupet C-A, Ray A, Tupin E, et al. A novel MVA-based multiphasic vaccine for prevention or treatment of tuberculosis induces broad and multifunctional cell-mediated immunity in mice and primates. PLoS One. 2015:10:e0143552.

10. Dai L. Generation, Characterization and Application of a Novel BAC System for MVA Mutagenesis to Investigate the Function of Vaccinia Virus Immune Modulatory Gene N1L. Munich: Technische Universität München; 2014.

11. Cottingham MG, Gilbert SC. Rapid generation of markerless recombinant MVA vaccines by en passant recombineering of a self-excising bacterial artificial chromosome. J Virol Methods. 2010;168:233-6.

12. Wang Z, Martinez J, Zhou W, La Rosa C, Srivastava T, et al. Modified H5 promoter improves stability of insert genes while maintaining immunogenicity during extended passage of genetically engineered MVA vaccines. Vaccine. 2010;28:1547-57.

13. Nagel $\mathrm{C}-\mathrm{H}$, Pohlmann A, Sodeik B. Construction and characterization of bacterial artificial chromosomes (BACs) containing herpes simplex virus fulllength genomes. Methods Mol Biol. 2014;1144:43-62.

14. Rocha CD, Caetano BC, Machado AV, Bruña-Romero O. Recombinant viruses as tools to induce protective cellular immunity against infectious diseases. Int Microbiol. 2004;7:83-94

15. Bertolotti-Ciarlet A, White $\sqcup$, Chen R, Prasad BV, Estes MK. Structural requirements for the assembly of Norwalk virus-like particles. J Virol. 2002;76:4044-55.

16. Draper SJ, Heeney JL. Viruses as vaccine vectors for infectious diseases and cancer. Nat Rev Microbiol. 2010;8:62.

17. Hardy ME, White LJ, Ball JM, Estes MK. Specific proteolytic cleavage of recombinant Norwalk virus capsid protein. J Virol. 1995;69:1693-8.

18. Ausar SF, Foubert TR, Hudson MH, Vedvick TS, Middaugh CR. Conformational stability and disassembly of Norwalk virus-like particles effect of pH and temperature. J Biol Chem. 2006;281:19478-88.

19. Gudmundsdotter L, Nilsson C, Brave A, Hejdeman B, Earl P, et al. Recombinant modified vaccinia Ankara (MVA) effectively boosts DNAprimed HIV-specific immune responses in humans despite pre-existing vaccinia immunity. Vaccine. 2009;27:4468-74.

20. Cooney E, Collier A, Greenberg P, Coombs R, Zarling J, et al. Safety of and immunological response to a recombinant vaccinia virus vaccine expressing HIV envelope glycoprotein. Lancet. 1991:337:567-72.

21. Ura T, Okuda K, Shimada M. Developments in viral vector-based vaccines. Vaccines. 2014:2:624-41.

22. Gómez CE, Perdiguero B, Cepeda MV, Mingorance L, García-Arriaza J, et al. High, broad, polyfunctional and durable $T$ cell immune responses induced in mice by a novel hepatitis C virus ( $\mathrm{HCV}$ ) vaccine candidate based on MVA expressing the near full-length HCV genome (MVA-HCV). J Virol. 2013;87(13): 7282-300.

23. Mooij P, Balla-Jhagjhoorsingh SS, Koopman G, Beenhakker N, Van Haaften P, et al. Differential CD4+ versus CD8+ T-cell responses elicited by different poxvirus-based human immunodeficiency virus type 1 vaccine candidates provide comparable efficacies in primates. J Virol. 2008:82:2975-88.

24. Geiben-Lynn R, Greenland JR, Frimpong-Boateng K, Letvin NL. Kinetics of recombinant adenovirus type 5 , vaccinia virus, modified vaccinia Ankara virus, and DNA antigen expression in vivo and the induction of memory Tlymphocyte responses. Clin Vaccine Immunol. 2008;15:691-6.

25. Wyatt LS, Shors ST, Murphy BR, Moss B. Development of a replicationdeficient recombinant vaccinia virus vaccine effective against parainfluenza virus 3 infection in an animal model. Vaccine. 1996;14:1451-8.

26. Baur K, Brinkmann K, Schweneker M, Pätzold J, Meisinger-Henschel C, et al. Immediate-early expression of a recombinant antigen by modified vaccinia virus Ankara breaks the immunodominance of strong vector-specific B8R antigen in acute and memory CD8 T-cell responses. J Virol. 2010;84:8743-52.

27. Orubu T, Alharbi NK, Lambe T, Gilbert SC, Cottingham MG. Expression and cellular immunogenicity of a transgenic antigen driven by endogenous poxviral early promoters at their authentic loci in MVA. PLoS One. 2012:7:e40167.

28. Meisinger-Henschel C, Schmidt M, Lukassen S, Linke B, Krause L, et al. Genomic sequence of chorioallantois vaccinia virus Ankara, the ancestor of modified vaccinia virus Ankara. J Gen Virol. 2007;88:3249-59.

29. Meyer H, Sutter G, Mayr A. Mapping of deletions in the genome of the highly attenuated vaccinia virus MVA and their influence on virulence. J Gen Virol. 1991;72:1031-8.

30. Kremer M, Volz A, Kreijtz JH, Fux R, Lehmann MH, Sutter G. Easy and efficient protocols for working with recombinant vaccinia virus MVA. Methods Mol Biol. 2012;890:59-92.

31. Jamsai $D$, Orford $M$, Nefedov $M$, Fucharoen $S$, Williamson $R$, et al. Targeted modification of a human $\beta$-globin locus BAC clone using 
GET recombination and an I-Scel counterselection cassette. Genomics. 2003:82:68-77.

32. Moeini H, Omar AR, Rahim RA, Yusoff K. Development of a DNA vaccine against chicken anemia virus by using a bicistronic vector expressing VP1 and VP2 proteins of CAV. Comp Immunol Microbiol Infect Dis. 2011;34:227-36.

33. Tischer BK, Smith GA, Osterrieder N. En passant mutagenesis: a two step markerless red recombination system. Methods Mol Biol. 2010;634:421-30.

34. Yao X-D, Evans DH. High-frequency genetic recombination and reactivation of orthopoxviruses from DNA fragments transfected into leporipoxvirusinfected cells. J Virol. 2003;77:7281-90.

\section{Publisher's Note}

Springer Nature remains neutral with regard to jurisdictional claims in published maps and institutional affiliations.

Ready to submit your research? Choose BMC and benefit from:

- fast, convenient online submission

- thorough peer review by experienced researchers in your field

- rapid publication on acceptance

- support for research data, including large and complex data types

- gold Open Access which fosters wider collaboration and increased citations

- maximum visibility for your research: over $100 \mathrm{M}$ website views per year

At BMC, research is always in progress.

Learn more biomedcentral.com/submissions 